\title{
Heilige und vorletzte Wirklichkeit. Einsatz Johannes Pauls II. für die Würde des menschlichen Lebens
}

\author{
Sacred Reality, Penultimate Reality. \\ Commitment of John Paul II to the protection of the dignity of human life
}

\begin{abstract}
John Paul II could be rightly called the 'Admirer of life'. His deep faith, along with the existential reasons, the sources of which could be traced back to his traumatic experiences during the Second World War, moulded him into a strong defender of human life. The Pope teaches that a civilization's worth is determined by its attitude towards human life; it is either the civilization of life, or the destructive civilization of death. Since body constitutes an integral part of the whole human person, its dignity is enhanced. In his 'theology of the body' John Paul II stressed not only the sharing of the body in the dignity of the person, but also its importance as a sign and a needle in the compass of the human intellect in its search for moral norms. The Polish Pope insisted on the value of human life from its inception to natural death, and often presented various ruling elites with a challenge: Life is sacrosanct and this truth must be reflected in legislation. Human life is, according to John Paul II, the penultimate things; life is limited, mortal. The value of human life is determined by its destination: the human being is to find his fulfillment in eternity, where he will have a share in the everlasting life. Thirty years after the elevation of Karol Wojtyla to the Holy See we may confidently give him the credit for influencing the change in sensitivity to human life on a truly global scale. Though the contrary tendencies are very strong, thanks to the Polish Pope the impact of the culture of death has been significantly restricted.
\end{abstract}

\section{Keywords}

Anti-life mentality, civilization of death, dignity, value of life, sacredness of life, death, dying. 
Alle Päpste des letzten Jahrhunderts waren zweifelsohne große Persönlichkeiten. Die Vor- und Nachkriegszeiten mit ihren Turbulenzen und ihrer Dramatik sowie nicht zuletzt die kulturellen Umwälzungen der zweiten Hälfte des 20. Jahrhunderts verbunden mit der Konzilsperiode in der Kirche haben den Dienst der Nachfolger Petri tief geprägt. Die Gestalt Johannes Pauls II. ragt in ihrer Aufzählung nicht nur durch die Länge seines Pontifikats heraus, sondern vor allem durch seinen unermüdlichen Einsatz für den Lebensschutz. Man könnte ihn sicher charakterisieren als den Papst der Menschenwürde, den Papst des Lebens. Schon in seiner ersten Enzyklika Redemptor hominis (1979) hat er in einer programmatischen Weise die Richtung seines Einsatzes und gleichzeitig die spezifische Akzentuierung seines Pontifikats vorgezeichnet. Obwohl sie eigentlich eine christologische Enzyklika war, lenkte sie in besonderer Weise die Aufmerksamkeit auf die Inkarnation, die Menschwerdung des ewiges Logos und betraf auf diese Weise den Menschen und die Würde seines Lebens.

Der Einsatz Johannes Pauls II. für die Würde des menschlichen Lebens umfasst nicht nur partikuläre Aktionen und Interventionen, sondern wesentlich auch eine Intensivierung und Vertiefung der theologischen und anthropologischen Reflexion. Der Großteil seines imposanten Schrifttums bezieht sich auf die Lebensproblematik, zahlreiche Äußerungen behandeln direkt die Frage des Lebensschutzes. Nicht weniger bedeutend waren jedoch die zahlreichen Initiativen des polnischen Papstes auf der institutionellen Ebene. Unter den neuen institutionellen Initiativen war u.a. 1985 die Errichtung der Päpstlichen Kommission für die Pastoral im Krankendienst, die 1988 zum Rang des Päpstlichen Rates erhoben wurde (zum Präsident dieses Rates wurde 2009 vom Papst Benedikt XVI. der polnische Erzbischof Zygmunt Zimowski ernannt). Eine der wichtigsten Initiativen zum Schutz des Lebens war 1994 die Errichtung der Päpstlichen Akademie für das Leben, bekannt unter ihrem lateinischen Namen „Pro Vita“. Es war eine adäquate Antwort auf die Intensivierung der bioethischen Diskussion in der Welt, die angesichts des rasanten Fortschritts in der Biomedizin auch die gravierenden Unterschiede in der ethischen Beurteilung einiger medizinischer Prozeduren aufdeckte. $\mathrm{Zu}$ den wichtigsten Aufgaben der Akademie, deren Mitglieder namhafte Theologen, Bioethiker und Mediziner sind, gehört bis heute die Förderung der christlichen Lebensethik.

Die folgenden Analysen werden sich nur am Rande mit päpstlichen Äußerungen zu partikulären bioethischen Themen befassen, wenngleich solche auch erwähnt werden müssen. Es wird eher um das zentrale Grundthema des Lebens gehen. Die Antwort auf ethische Einzelfragen hängt wesentlich von der Sicht des Menschen $\mathrm{ab}$, von der anthropologischen Grundkonzeption, deren wichtiger Bestandteil die 
Begründung für die Würde des menschlichen Lebens ist. In vier Schritten werden zuerst die wesentliche Bedeutung der menschlichen lebendigen Leiblichkeit und dann der hohe Wert des Lebens sowie ihre Begrenzung und die Hoffnung auf die ewige Vollendung behandelt.

\section{Leibliches Leben}

Auf den ersten Blick kann man den Eindruck haben, dass die gegenwärtige Mentalität die menschliche Leiblichkeit hochschätzt und der leiblichen Dimension des Menschen ihre Bedeutung zurückgibt. Dieser Eindruck täuscht jedoch. Man kann zwar im Bereich der Kunst und der Medien eine Konzentration auf die materielle Komponente des Menschen beobachten, sie geht jedoch mit einer reduktiven Sicht des Menschen einher. Der Leib ist nicht mehr ein wesentlicher Aspekt der menschlichen Natur. Er wird zum Gegenstand, zum Körper in der Welt anderer Körper, der von der freien Vernunft als Objekt behandelt und manipuliert werden kann und darf. Eine solche Einstellung scheint eine Spielart dualistischen Denkens zu sein, welche seit Descartes und Julien de la Mettrie das Wesentliche im Menschen lediglich in seiner Vernunft erblickt und seine materielle Komponente ganz in die Welt der Materie verweist und ihn als eine lebendige Maschine betrachtet.

Johannes Paul II. hat schon in den Anfängen seines Pontifikats in Mittwochskatechesen ${ }^{1}$ die hohe Bedeutung des menschlichen Leibes gewürdigt. In seinem Projekt einer „Theologie des Leibes“, durchdacht und in groben Zügen noch in seiner Krakauer Zeit entworfen, vertieft der Papst die Konzilsbezeichnung der menschlichen Natur als corpore et anima unus ${ }^{2}$. Der menschliche Leib ist kein materieller „Zusatz“, sondern eine wesentliche Dimension der menschlichen Natur. Diese Feststellung wird gravierende Konsequenzen bei der Lösung vieler bioethischen Kontroversen haben. Vorausgesetzt, dass das Wesen des Menschen sich in seinen geistigen Fähigkeiten erschöpft, umfasst die zu schützende Menschenwürde den Leib jener Menschen nicht, die solche Fähigkeiten noch nicht aktualisieren können (Embryonen, Föten) sowie jener, die sie endgültig verloren haben (z.B. dauerhaft Bewusstlose). Das Leben derartiger „menschlicher Hüllen“, wie es die Befürworter einer solchen Meinung bezeichnet, darf beendet

\footnotetext{
${ }^{1}$ Sie wurden in der Zeit von 1979 bis 1984 gehalten und dann in einem Band herausgegeben unter dem Titel: Johannes Paul II., Die menschliche Liebe im göttlichen Heilsplan: eine Theologie des Leibes, Mittwochskatechesen von 1979-1984, Kisslegg 2008.
}

${ }^{2}$ Gaudium et spes 14. 
werden und ihre Tötung wird weder als Mord bezeichnet noch geahndet. Der entschiedene Einsatz Johannes Pauls II. für den Schutz des Menschen von der Empfängnis bis zum natürlichen Tod fußt dagegen auf einer uneingeschränkten Hochschätzung des menschlichen Leibes. Solange der Leib des Menschen aus eigener Kraft lebendig bleibt, lebt die menschliche Person, selbst wenn sie das Bewusstsein und den Kontakt zur Umwelt auf Dauer verloren hat.

Die Thematik des Leibes ist in der bioethischen Diskussion nicht nur am Anfang und am Ende des Lebens von Bedeutung, sondern auch dort, wo der Mensch grundsätzlich nach der moralischen Orientierung sucht. Die fundamentale Einheit des Menschen begründet nicht nur die hohe Würde der menschlichen Leiblichkeit und somit der leiblichen Dimension des Lebens, sondern verweist auch auf den richtigen Gebrauch der Dynamik, die sich im lebendigen menschlichen Leib manifestiert. In seiner Moralenzyklika Veritatis splendor (1993) verweist Johannes Paul II. auf einige gegenwärtige ethische Tendenzen, die die Bedeutung und den Wert des menschlichen Leibes reduzieren. Es ist eine verabsolutierte Freiheit, die ,schließlich den menschlichen Leib wie Rohmaterial [behandelt], bar jeglichen Sinnes und moralischen Wertes, solange die Freiheit es nicht in ihr Projekt eingebracht hat. Die menschliche Natur und der Leib erscheinen folglich als für die Wahlakte der Freiheit materiell notwendige, aber der Person, dem menschlichen Subjekt und der menschlichen Handlung äußerliche Voraussetzungen oder Bedingtheiten "3. Eine solche Sicht spaltet den Menschen in zwei Teile: während die Dynamismen der materiellen, körperlichen Komponente der menschlichen Natur höchstens physische, also vor-sittliche Güter bezwecken, kann nur die freie Vernunft sittliche Zwecke bestimmen und moralische Normen formulieren. Demgegenüber betont der Papst, dass die menschliche Person gerade ,in ihrem Leib die vorwegnehmenden Zeichen, den Ausdruck und das Versprechen der Selbsthingabe in Übereinstimmung mit dem weisen Plan des Schöpfers [entdecken kann] ${ }^{\star 4}$.

\section{Heiliges Leben}

Das Zweite Vatikanische Konzil bestätigt die Intuition des Menschen, der sich auf Grund seiner besonderen Fähigkeiten über alle anderen Lebewesen erhaben fühlt ${ }^{5}$. Diese besondere Stellung, den erhabenen Status des Menschen

\footnotetext{
${ }^{3}$ Johannes Paul II., Enzyklika Veritatis splendor 48.

${ }^{4}$ Johannes Paul II., Enzyklika Veritatis splendor 48.

${ }^{5}$ Gaudium et spes 14.
} 
in der gesamten Schöpfung hat schon Immanuel Kant in seinem kategorischen Imperativ formuliert. Während er den Status der Dinge und anderer Lebewesen als Wert bezeichnet, verwendet er für die Beschreibung der Stellung des Menschen den Begriff der Würde ${ }^{6}$. Obwohl die philosophischen Wurzeln Karol Wojtyłas nicht in der kantischen Philosophie lagen, formulierte er in einer sprachlichen Anlehnung an Kant die sog. ,personalistische Norm“. Sie besagt, dass eine Person ein solches Gut ist, das niemals bloß in Form eines Gebrauchsgegenstandes als Mittel zum Zweck gebraucht werden darf. Die einzig richtige Einstellung zu ihr ist die Liebe 7 . Diesem Grundsatz blieb Johannes Paul II. während seines gesamten Pontifikats treu, aus ihm resultiert auch die päpstliche Überzeugung von der Heiligkeit des menschlichen Lebens. Sie wurde gleich am Anfang einer der wichtigsten Enzykliken Johannes Pauls II., Evangelium vitae (1995) feierlich proklamiert. Das menschliche Leben ist zweifellos eine „heilige Wirklichkeit, die uns anvertraut wird, damit wir sie mit Verantwortungsgefühl hüten und in der Liebe und Selbsthingabe an Gott sowie an die Schwestern und Brüder zur Vollendung bringen " ${ }^{\text {}}$. Mehrmals in seinen Äußerungen betont der Papst, dass die Heiligkeit des Lebens und die daraus resultierende höchste Achtung, die ihm gebührt, die ganze Zeitspanne des Lebens umfasst, also von der Empfängnis bis zum natürlichen Tod reicht ${ }^{9}$.

${ }^{6}$ Vgl. I. Kant, Grundlegung der Sitten, Stuttgart 1991, S. $87 \mathrm{f}$.

${ }^{7}$ K. Wojtyła, Miłość i odpowiedzialność, Lublin 2001, S. 42.

${ }^{8}$ Johannes Paul II., Enzyklika Evangelium vitae 2. Es lohnt sich zu bemerken, wie stark die Ablehnung der Abtreibung und der Euthanasie vom Papst hier betont wird. Obwohl eine Enzyklika an sich nicht zu unfehlbaren Äußerungen des Papstes gehört, zählt Johannes Paul II. bei der Ablehnung der Abtreibung und der Euthanasie die Bedingungen auf, welche die höchstverbindliche päpstliche Verkündigung markieren: „Mit der Autorität, die Christus Petrus und seinen Nachfolgern übertragen hat, erkläre ich deshalb in Gemeinschaft mit den Bischöfen - die mehrfach die Abtreibung verurteilt und, obwohl sie über die Welt verstreut sind, bei der eingangs erwähnten Konsultation dieser Lehre einhellig zugestimmt haben - dass die direkte, das heißt als Ziel oder Mittel gewollte Abtreibung immer ein schweres sittliches Vergehen darstellt, nämlich die vorsätzliche Tötung eines unschuldigen Menschen. Diese Lehre ist auf dem Naturrecht und auf dem geschriebenen Wort Gottes begründet, von der Tradition der Kirche überliefert und vom ordentlichen und allgemeinen Lehramt der Kirche gelehrt“ (Johannes Paul II., Enzyklika Evangelium vitae 62; vgl. auch Johannes Paul II., Enzyklika Evangelium vitae 57).

${ }^{9}$ Vgl. Johannes Paul II., Apostolisches Schreiben Novo Millennio inneunte 51, in: http:// www.vatican.va/holy_father/john_paul_ii/apost_letters/documents/hf_jp-ii_apl_20010106_novomillennio-ineunte_ge.html (16.03.2012): „Ein besonderes Engagement muss einigen Aspekten der Radikalität des Evangeliums gelten, die oft so wenig verstanden werden, dass sie die Intervention der Kirche unpopulär machen, die aber deshalb in der kirchlichen Agenda der Liebe nicht weniger präsent sein dürfen. Ich beziehe mich auf die Verpflichtung, sich für die Achtung des Lebens eines jeden Menschen von der Empfängnis bis zu seinem natürlichen Hinscheiden einzusetzen“. 
Das Prinzip der Heiligkeit des menschlichen Lebens markiert einen der schärfsten Gegensätze in der bioethischen Debatte. Dieser christlichen Überzeugung wird in der gesellschaftlichen Diskussion das Prinzip der Lebensqualität gegenübergestellt. Das menschliche Leben ist danach grundsätzlich nicht sakrosankt, es hat nur dann einen Wert, der seine Kontinuität rechtfertigt, wenn es eine entspreche Qualität aufweist. Es ist verständlich, dass diese im Rahmen des vorherrschenden Relativismus und Subjektivismus nur von Fall zu Fall, eben subjektiv bestimmt werden darf. Jeder einzelne Betroffene muss selbst entscheiden, ob sein Lebensniveau entsprechende Qualitätsmerkmale aufweist. Diese vordergründig die Autonomie des einzelnen aufwertende Einsicht kann zu gravierenden Konsequenzen führen. Die geringe Selbsteinschätzung vieler schwerstkranker und behinderter Menschen wird unter diesen Voraussetzungen leicht in eine Bitte um Euthanasie einmünden. Statt der Unterstützung und Solidarität der Umgebung und der mitmenschlichen Hilfe im würdigen Tragen der Lebenslast, werden solche Personen sich selbst überlassen und noch zusätzlich von einer Gesellschaft, die das schwerstkranke Leben als allgemein lebensunwürdig einzuschätzen scheint, unter Druck gestellt. Solche Ansichten sind charakteristisch für eine Mentalität, die Johannes Paul II. als anti-life mentality bezeichnet. Ihre Popularisierung führt zur Entstehung einer Kultur des Todes und, wenn sie in politische Programme und rechtliche Initiativen eindringt, kann die ganze Zivilisation in Richtung einer Zivilisation des Todes umkippen ${ }^{10}$.

\section{Begrenztes Leben}

Die hohe Einschätzung und die Verpflichtung zum Schutz des menschlichen Lebens bedeutet nicht, dass es einen absoluten Wert darstellt. Johannes Paul II. bedient sich eines Neologismus und stellt fest, dass das Leben des Menschen „,in Wahrheit [...] nicht »letzte«, sondern »vorletzte« Wirklichkeit"11 ist. Dies

${ }^{10}$ Vgl. Johannes Paul II, Brief an die Familien 13, in: http://www.vatican.va/holy_father/john paul_ii/letters/documents/hf_jp-ii_let_02021994_families_ge.html (16.03.2012): „Die Zivilisation der Liebe ruft Freude hervor: unter anderem Freude darüber, dass ein Mensch zur Welt kommt (vgl. Joh 16,21), und folglich, weil die Gatten Eltern werden. Zivilisation der Liebe bedeutet »sich an der Wahrheit freuen « (vgl. 1 Kor 13,6). Aber eine Zivilisation, die sich an einer konsumistischen und geburtenfeindlichen Gesinnung inspiriert, ist keine Zivilisation der Liebe und kann es niemals sein“. Vgl. auch Johannes Paul II., Enzyklika Evangelium vitae 21-24.

${ }^{11}$ Ibidem. Vgl. auch Johannes Paul II., Brief an die alten Menschen, 16, in: http://www. vatican.va/holy_father/john_paul_ii/letters/documents/hf_jp-ii_let_01101999_elderly_ge.html (16.03.2012): „Die Beispiele [der Heiligen - Einf. M.M.] werfen keinerlei Schatten auf den Wert 
bedeutet nicht nur, dass es für höhere Werte, wie z.B. für den religiösen Glauben bzw. auf Grund der Treue zu seinem Gewissen geopfert werden darf, sondern impliziert auch seine Vergänglichkeit ${ }^{12}$.

Das menschliche Leben ist begrenzt, vergänglich, sterblich. Der Tod ist nicht ein Sonderfall, der nur einige Menschen betrifft, die z.B. durch einen tragischen Unfall, in Folge einer unerwarteten Krankheit bzw. als selbst gewähltes Schicksal sterben. Die Sterblichkeit gehört wesentlich zur Kontingenz der menschlichen Natur. Deswegen ist die Annahme des Todes als letzter Lebensakt, der - wie das ganze Leben - würdig erlebt werden muss, auch eine Form der Lebensbejahung. Da die meisten Menschen in der letzten Zeitspanne ihres Lebens auf die Hilfe der Medizin angewiesen sind, gehört die Hilfe bei der Akzeptanz der Sterblichkeit zu den wichtigen Aufgaben der Ärzte. Wie Johannes Paul II. in einer Ansprache an die Ärzte betont, muss man „sich um die Kranken kümmern mit einem gesunden Realismus, der es vermeidet, im Leidenden die Illusion von der Allmacht der Medizin zu wecken. Es gibt Grenzen, die der Mensch nicht überwinden kann; in solchen Fällen muss man den eigenen, menschlichen Zustand, den der Gläubige im Licht des göttlichen Willens zu deuten vermag, mit Gelassenheit akzeptieren. Dieser Wille offenbart sich auch im Tod, dem natürlichen Zielpunkt des Lebens auf Erden. Es ist Teil eurer Aufgabe, die Menschen zur unbesorgten Annahme des Todes zu erziehen" ${ }^{\prime 13}$.

Es ist schon bemerkenswert, dass gerade dieser Papst, der so viel für den Schutz und die Hochschätzung des Lebens getan hat, sich auch vehement für ein würdiges Sterben des Menschen einsetzte. Und dieser Einsatz Johannes Pauls II. wirkte am eindrucksvollsten auf die Weltöffentlichkeit. Der polnische Papst, der es wie kaum ein Kirchenvertreter verstand, die mediale Präsenz des Evangeliums zu intensivieren, vollendete sein Leben auch vor den Augen der Millionen. Seine

des irdischen Lebens, das trotz Einschränkungen und Leiden schön ist und bis zum Ende gelebt werden muss. Sie erinnern uns jedoch daran, dass dieses Leben nicht der letzte Wert ist, dass also nach christlicher Auffassung der Lebensabend die Konturen eines »Überganges« annimmt, einer von einem Leben zum anderen geschlagenen Brücke zwischen der zerbrechlichen und unsicheren Freude dieser Erde und der vollkommenen Freude, die der Herr seinen treuen Dienern bereitet: "Komm, nimm teil an der Freude deines Herrn!“ (Mt 25, 21)“.

${ }^{12}$ Johannes Paul II., Enzyklika Evangelium vitae 47: „Sicher ist für den Gläubigen das physische Leben in seinem irdischen Zustand kein Absolutum, so das von ihm gefordert werden kann, es um eines höheren Gutes willen aufzugeben; denn, wie Jesus sagt, »wer sein Leben retten will, wird es verlieren; wer aber sein Leben um meinetwillen und um des Evangeliums willen verliert, wird es retten« $(\mathrm{Mk} 8,35) “$.

13 Johannes Paul II., Audienz für die Teilnehmer am Internationalen Kongress über Gastroenterologie(23.03.2002), in:http://www.vatican.va/holy_father/john_paul_ii/speeches/2002/ march/documents/hf_jp-ii_spe_20020323_congr-gastroenterologia_ge.html (16.03.2012). 
letzten Tage und Stunden wurden als eine tiefgründige Katechese über die Würde des Sterbens und die Würde des sterbenden Menschen bezeichnet. Und dies nicht nur aus dem Grund, dass es dabei um eine herausragende Persönlichkeit ging, um einen Menschen, dessen Beerdigung mit dem lautstarken Ruf der Glaubenden „Santo Subito“ begleitet wurde. Sein Sterben illustrierte eindrucksvoll seine zahlreichen Äußerungen über den Wert des hohen Alters und die Wichtigkeit einer würdevollen Gestaltung des Lebensendes, soweit das in menschlicher Macht steht. Zum würdigen Sterben gehört ein entschiedenes „Nein“ zur Euthanasie in irgendeiner Form, das der Papst oft und eindeutig geäußert hat. Obwohl sie manchmal mit guter Absicht durchgeführt wird, indem man dem grausamen Leiden eines Schwerstkranken ein Ende setzen will, bleibt die Euthanasie immer ein in sich schlechter Akt, eine Beleidigung der Menschenwürde ${ }^{14}$. Johannes Paul II. betonte jedoch, dass dieser Einsatz für das Leben bis zuletzt nicht in therapeutischen Übereifer überschlagen darf. Eine aggressive Behandlung angesichts des sicheren Todes, die ihren medizinischen Sinn verloren hat und nur zusätzliche Schmerzen bereitet, darf abgebrochen werden. Der Papst erinnert daran, dass das Sittengesetz den Verzicht auf sogenannten „«therapeutischen Übereifer» billigt und nur jene Behandlungen verlangt, die zu den normalen Erfordernissen ärztlicher Betreuung gehören" ${ }^{\text {"15 }}$.

Ein christlich gelebtes Bewusstsein der Vergänglichkeit und Sterblichkeit des Lebens muss also von der Überzeugung begleitet werden, dass der Wert des Lebens nicht an der körperlichen Gesundheit, am Maß der physischen Kräfte und an den mentalen Fähigkeiten gemessen werden darf. Das Leben ist lebenswert bis zum letzten Atemzug. Gleichzeitig jedoch ermöglicht gerade der christliche Glaube den Ausblick über den Tod hinaus. Der Christ lebt nicht nur im Bewusstsein des sicheren Todes, sondern in der begründeten Hoffnung der ewigen Vollendung. Diese Hoffnung drückt der hochbetagte Papst wenige Jahre vor seinem Tod (1999) im Brief an seine „Schwestern und Brüder im hohen Alter“ aus: „Trotz der Einschränkungen, die mit dem Alter verbunden sind, bewahre ich mir die Lebensfreude. Dafür danke ich dem Herrn. Es ist schön, sich bis zum Ende für die Sache des Reiches Gottes zu verzehren. Gleichzeitig empfinde ich einen großen Frieden, wenn ich an den Augenblick denke, in dem der Herr mich zu sich rufen wird: vom Leben ins Leben!" ${ }^{16}$.

\footnotetext{
${ }^{14}$ Johannes Paul II., Enzyklika Evangelium vitae 65.

${ }^{15}$ Johannes Paul II., Brief an die alten Menschen 9.

${ }^{16}$ Johannes Paul II., Brief an die alten Menschen 17.
} 


\section{Ewiges Leben}

Die christliche Anthropologie bestreitet keineswegs die im Bereich der Ethik vorherrschende Begründung der Menschenwürde, die an besondere geistige Fähigkeiten der menschlichen Person anknüpft. Durch seine Vernunft, seine Freiheit und die damit verbundene Fähigkeit der Selbstbestimmung überschreitet der Mensch alle anderen Geschöpfe und genießt deswegen einen besonderen Status. Theologisch gesehen entsprechen diese Grunddaten der Schöpfung des Menschen als Abbild Gottes, imago Dei. Diese Feststellungen sind jedoch in der theologischen Anthropologie keineswegs der Abschluss, sondern eher der Ausgangspunkt der Reflexion. Die besondere Würde des Menschen wird im Rahmen des Erlösungswerkes Christi noch mehr betont. Die Inkarnation des ewigen Wortes und die durch ihn vollzogene Erlösung der menschlichen Natur verleiht der Person einen unvergleichlichen Wert. Das theologische Nachdenken über das menschliche Leben mündet in seine Bestimmung für das ewige Leben. Das menschliche Leben wird verwandelt, erlischt jedoch nicht im Tod ${ }^{17}$.

Johannes Paul II. betonte diese Überzeugung der Kirche oft, vor allem dort, wo im Rahmen der bioethischen Reflexion dem menschlichen Leben aufgrund seiner Unvollkommenheit die Würde abgesprochen wird. Die Ewigkeitsperspektive bewahrt vor der Geringschätzung des Lebens solcher Menschen. „Der Mensch ist zu einer Lebensfülle berufen, die weit über die Dimensionen seiner irdischen Existenz hinausgeht, da sie in der Teilhabe am Leben Gottes selber besteht. Die Erhabenheit dieser übernatürlichen Berufung enthüllt die Größe und Kostbarkeit des menschlichen Lebens auch in seinem zeitlich-irdischen Stadium" "18.

Letztendlich ist es gerade diese Perspektive der ewigen Vollendung, die das Leben des Menschen als ausschließlich unter der göttlichen Verfügungsmacht stehendes Gut zu verstehen ermöglicht. Der Herr des Lebens ist allein Gott. „Das menschliche Leben ist als etwas Heiliges anzusehen, da es ja schon von seinem Anfang an das Handeln des Schöpfers erfordert und immer in einer besonderen Beziehung mit dem Schöpfer, seinem einzigen Ziel, verbunden

17 Johannes Paul II, Apostolisches Schreiben Salvifici doloris 15: „Infolge des Heilswerkes Christi lebt der Mensch auf Erden in der Hoffnung auf das ewige Leben und die ewige Heiligkeit. Und wenn auch der von Christus durch Kreuz und Auferstehung vollbrachte Sieg über Sünde und Tod die irdischen Leiden aus dem Leben des Menschen nicht hinwegnimmt und auch nicht die ganze geschichtliche Dimension des menschlichen Daseins vom Leiden befreit, so wirft er doch auf diese Dimension insgesamt und auf jedes einzelne Leiden ein neues Licht, das Licht der Erlösung“"

${ }^{18}$ Johannes Paul II., Enzyklika Evangelium vitae 2. 
bleibt. Gott allein ist der Herr des Lebens vom Anfang bis zum Ende“19. Ohne die Perspektive der ewigen Vollendung wäre es unmöglich, dem Leiden, das in verschiedenem Maß mit jedem menschlichen Leben verbunden ist, einen Sinn zu verleihen. Wenngleich das Leiden und der Schmerz immer sehr vielschichtige Wirklichkeiten sind, können sie im Licht des Evangeliums zur vertieften Sicht des Lebenssinns beitragen. Auch das Leben eines Menschen, das von einem chronischen Leiden gezeichnet ist, ist in der Ewigkeitsperspektive nicht sinnlos ${ }^{20}$.

\section{Abschluss}

In seinem Einsatz für die Würde des menschlichen Lebens machte Johannes Paul II. die Einstellung zum Leben zum Maßstab der Zivilisation. Vor allem in seinen fragilsten Phasen, d.h., an seinem Anfang und Ende, ist das Leben des einzelnen Menschen auf den Schutz und die Unterstützung seiner Umgebung angewiesen. Innerhalb der westlichen Kultur erblickte der Papst starke und einflussreiche Tendenzen, die zur Etablierung einer Zivilisation des Todes führen können. Sie werden vor allem in den offensiv betriebenen Vorhaben sichtbar, Abtreibung und Euthanasie zuerst zu depönalisieren, dann zu legalisieren und letztendlich zum Rang eines Menschenrechtes zu erheben. Der entschiedene Einsatz des Papstes galt der Etablierung einer Kultur der Achtung vor jedem menschlichen Leben und dadurch einer Zivilisation der Liebe. Die zahlreichen Äußerungen, darunter auch Stellungnahmen und Appelle an die Regierenden, aber vor allem die starke Persönlichkeit des Papstes, weckten viele Gewissen zur eindeutigen und entschiedenen Parteinahme für das menschliche Leben. Diese Problematik markiert auch die Hauptlinie der Kritik am Papst seitens jener, die den Wert des Menschen nicht am bloßen Faktum seiner Existenz, sondern an der aktuell vorhandenen Qualität seines Lebens messen.

19 Johannes Paul II., Enzyklika Evangelium vitae 53. Der Papst zitiert hier die 1986 herausgegebene Instruktion der Kongregation für die Glaubenslehre Donum vitae.

${ }^{20}$ Johannes Paul II., Audienz für die Mitglieder der italienischen Liga für den Kampf gegen Tumorerkrankungen (25.02.2002), 3, in: http://www.vatican.va/holy_father/john_paul_ii/ speeches/2002/february/documents/hf_jp-ii_spe_20020225_lega-tumori_ge.html (16.03.2012). 